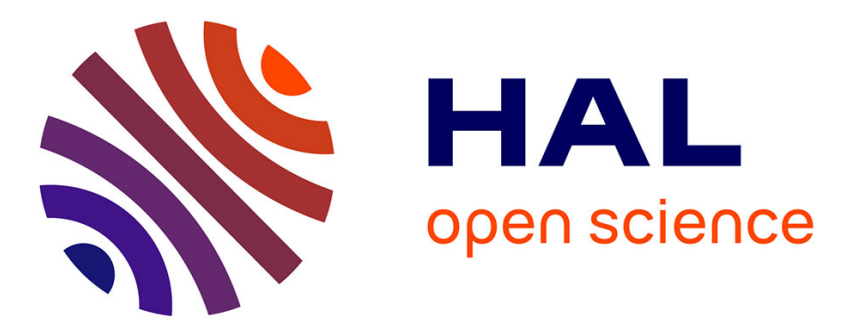

\title{
Du mouvement sans sens ou du sens sans mouvement: rôle des finalités et des contextes dans l'étude de comportements moteurs
}

\author{
Blandine Bril, Rémi Goasdoué
}

\section{- To cite this version:}

Blandine Bril, Rémi Goasdoué. Du mouvement sans sens ou du sens sans mouvement : rôle des finalités et des contextes dans l'étude de comportements moteurs. Intellectica - La revue de l'Association pour la Recherche sur les sciences de la Cognition (ARCo), 2009, 1 (59). halshs-01382966

\section{HAL Id: halshs-01382966 \\ https://shs.hal.science/halshs-01382966}

Submitted on 17 Oct 2016

HAL is a multi-disciplinary open access archive for the deposit and dissemination of scientific research documents, whether they are published or not. The documents may come from teaching and research institutions in France or abroad, or from public or private research centers.
L'archive ouverte pluridisciplinaire HAL, est destinée au dépôt et à la diffusion de documents scientifiques de niveau recherche, publiés ou non, émanant des établissements d'enseignement et de recherche français ou étrangers, des laboratoires publics ou privés. 


\section{Du mouvement sans sens ou du sens sans mouvement : rôle des finalités et des contextes dans l'étude de comportements moteurs}

\section{Blandine Bril ${ }^{1}$ \& Rémi Goasdoué ${ }^{2}$}

${ }^{1}$ Groupe de Recherche Apprentissage et Contexte, Ecole des Hautes Etudes en Sciences Sociales, 54 Bd Raspail, 75006 Paris

${ }^{2}$ Laboratoire EDA, Université Paris-Descartes, Rue des Saints Pères, 75006 Paris

blandine.bril@ehess.fr

remi.goasdoue@ehess.fr

Intellectica

Version révisée, mars 2009 


\section{Résumé}

Par leur richesse méthodologique et la diversité des activités qu'ils abordent, les travaux de E.J. Marey auraient pu être le point de départ de recherches conjuguant les exigences de contrôle expérimental reconnues aux sciences de la vie et la pertinence à l'égard des situations de terrain qu'offrent les sciences humaines. Cependant l'étude du mouvement demeure clivée entre des travaux privilégiant l'étude du sens, de la finalité ou de la fonction sociale des mouvements ou des recherches en sciences du mouvement recourant souvent à des mouvements sans sens, dénués de fonctionnalité. Quatre exemples volontairement éclectiques (marche, taille de la pierre, jeu du piano et du violon) nous permettront de montrer le rôle primordial du contexte de l'action et de la finalité dans l'organisation des comportements à travers deux types d'interactions : celles du corps et de l'environnement et celles du sujet manipulant un outil. Ces recherches tracent la voie d'une approche pluridisciplinaire du mouvement qui permettrait de sortir des apories auxquelles conduit l'étude de sens sans mouvement et de mouvements sans sens.

\section{Mots clés}

Activités finalisées, apprentissage et développement moteur, contrôle moteur, expérimentation de terrain, Marey, Demenÿ 
A la mémoire d'Esther Thelen

\section{"Ce n'est pas dans les laboratoires ordinaires de physiologie que l'on peut étudier les mouvements » (Marey 1883 p. 227).}

Cette phrase, qui peut sembler polémique hors de son contexte est publiée en 1883, dans un article de présentation de la station physiologique et des recherches qui y seront menées. Elle reflète d'une part l'intérêt de E.J. Marey pour ce que nous appelons des activités finalisées (Connolly \& Dalgleish 1989) comme la locomotion ou l'utilisation d'outils et, d'autre part les limites imposées par les expérimentations de laboratoire. Constatant les évolutions dans d'autres disciplines et sans mettre en cause l'apport des études de laboratoire à la physiologie, E.J. Marey (1883, op. cit.) considère que ces méthodes ne permettent pas d'accéder « au jeu de la vie normale ». La physiologie doit selon lui sortir du laboratoire et s'intéresser « aux différents problèmes de la vie pratique » sous peine de se voir " arrêter si l'on ne peut aller observer la nature dans son propre domaine » (Marey 1883, p. 226). Ces études in vivo restent cependant étroitement tributaires des techniques d'observation. Ainsi seule l'invention et le perfectionnement continuels des différentes méthodes graphiques et spécialement de la chronophotographie, ont rendu ce projet viable. L'intrication permanente des questionnements scientifiques et des évolutions technologiques fut à cette époque à son comble, l'innovation technique s'accompagna en effet d'un renouvellement des objets de recherche.

En fervents partisans de la méthode expérimentale, tout en ayant le souci de l'étude " grandeur nature », E.J. Marey et G. Demenÿ ont multiplié les comparaisons, filmant ainsi des hommes marchant, courant, sautant, combattant au bâton, à l'épée ou au poing, ou encore jouant du violon mais aussi une quantité considérable d'animaux, de l'insecte à l'éléphant en passant par le renard. Sans doute suscitée par l'enthousiasme de la puissance descriptive de la chronophotographie, la profusion d'images et l'éclectisme des espèces et des activités abordées révèlent néanmoins la volonté d'une démarche comparative (Marey 1883). Les études sur la locomotion humaine avaient ainsi pour but, à travers l'étude de différents contextes, de "chercher les conditions extérieures qui modifient ces actes, celles, par exemple, qui augmentent la vitesse de l'allure ou la longueur du pas et qui exercent ainsi une influence favorable ou défavorable sur la locomotion de l'homme » (Marey 1883, p. 228). Ce jeu sur les forces externes (Demenÿ 1924) qui contribuent à structurer les comportements, montre clairement qu'en sortant du laboratoire la physiologie, devient clairement une étude de l'interaction, et plus seulement une analyse du sujet isolé. Cette approche s'inscrit et étend la démarche fondatrice de C. Bernard, qui recommandait de se méfier de la physiologie des préparations isolées. L'esprit des travaux de E.J. Marey et son préparateur, G. Demenÿ auraient pu jeter les bases de recherches conjuguant les exigences de contrôle expérimental reconnues des sciences de la vie et la pertinence à l'égard des situations de terrain qu'offrent les sciences humaines. Cependant l'étude du mouvement demeure clivée entre des travaux privilégiant l'étude du sens, de la finalité ou de la fonction sociale des mouvements et des recherches en sciences du mouvement recourant le plus souvent à des mouvements sans sens. 


\section{Oppositions entre sciences humaines et sciences de la vie : mouvements ou gestes techniques}

Dans la tradition des travaux de M. Mauss (1936), de A.G. Haudricourt (1987) ou de A. Leroi-Gourhan (1964), les analyses des gestes techniques issues des recherches en sciences humaines reposent le plus souvent sur des descriptions et typologies prenant en compte l'effet produit sur l'environnement, ou encore la fonction sociale, voire symbolique des mouvements. A l'opposée l'enjeu des recherches en sciences de la vie n'est pas de traiter ces "causes finales" du mouvement (Siguan 1980) mais plutôt de s'intéresser aux "causes efficientes ", aux mécanismes de production et de contrôle du mouvement lui-même, sans intérêt particulier pour la tâche/activité effectuée. Cette focalisation sur le processus au détriment du l'intérêt porté au produit du mouvement, à deux conséquences : dans un soucis de faciliter le contrôle expérimental, les sujets sont placés dans des conditions souvent abstraites des caractéristiques essentielles des contextes quotidiens d'action, minimisant par exemple l'effet des forces ou encore limitant artificiellement le nombre de degrés de liberté impliqués dans les mouvements. La seconde conséquence intimement liée à ce souci de contrôle expérimental, est l'absence de fonctionnalité évidente pour le sujet participant à l'expérience. Soucieux, de maintenir «toutes choses égales par ailleurs », les travaux expérimentaux proposent des tâches souvent inédites pour ne pas avoir à contrôler l'influence de l'expertise antérieure des sujets. Cette solution de la nouveauté, apparemment simple pour garantir l'homogénéité des groupes s'avère lourde de conséquence pour l'étude du comportement moteur. Peut-on construire des tâches pour lesquelles nous sommes tous novices au même titre. Car la dimension artificielle ou naturelle d'une situation expérimentale ne se décrète pas par des critères externes simples, mais elle dépend étroitement de l'expérience antérieure du sujet. Nous défendons une position en marge de l'opposition récurrente entre laboratoire et terrain en affirmant qu'il n'y a pas de mouvements artificiels, pas plus que de situations plus particulièrement écologiques, il existe seulement des tâches (ensemble but / conditions de réalisation) plus ou moins inédites pour un individu donné. L'apparente nouveauté que présentent les mouvements sollicités dans certaines situations n'est pas neutre. À la manière dont les psychométriciens se sont confrontés à la question des « culture-free tests » (Cole 2005), on peut s'interroger sur la possibilité de trouver des tâches motrices réellement nouvelles pour un individu. Ne vaut-il pas mieux en ce cas contrôler cette relation avec l'expérience antérieure en proposant des tâches finalisées, c'est-à-dire ayant un sens pour celui qui l'exécute?

\section{"Live movement is a ball of entangle interaction"}

Le rôle majeur que jouent à la fois la finalité de l'action et le contexte dans lequel elle prend place à été abordé presque simultanément dans des démarches convergentes bien qu'étant partiellement isolées. Le plan du premier chapitre d'un ouvrage de synthèse de G. Demenÿ (1924) illustre en effet clairement la formule : "Live movement is a ball of entangle interaction" proposée pratiquement au même moment par N. Bernstein \& E. Popova (1929, p. 12). Cette convergence tient probablement non seulement à des préoccupations similaires, par rapport à l'analyse du travail mais surtout à des choix techniques et méthodologiques. N. Bernstein, comme E. Marey et G. Demenÿ ont étudié le mouvement d'experts, dans des situations préexistantes à l'expérimentation, à l'aide d'outils techniques et de formalisation mathématiques permettant une analyse détaillée de la complexité des comportements moteurs.

En pionnier de la biomécanique (Pociello 1999), G. Demenÿ oppose dans les causes du mouvement, forces internes et externes, distinction reprise et poussée plus loin dans l'analyse 
par N. Bernstein (1929/2003). Par la modélisation mécanique il décrit l'action conjointe de l'activité musculaire et des effets de la gravité dans l'organisation du mouvement. Cette distinction, étayée par deux nombreux exemples d'activités quotidiennes, montre à quel point les forces nécessaires à la production de mouvements ne sauraient être assimilées aux seules contractions musculaires. Au terme d'une série d'exemples d'actions graduellement virtuoses, N. Bernstein aboutit à cette définition : "demand for dexterity is not in the movements themselves but in surrounding conditions » (Bernstein 1996, p. 23). La prise en compte du contexte n'est pas une simple garantie de validité expérimentale, mais une nécessité pour l'intelligibilité des comportements. En conséquence, l'action peut être définie comme une propriété émergente de l'interaction de trois ensembles de contraintes, le sujet, la tâche et l'environnement (Newell 1986, Bril 2002). De manière complémentaire on pourrait définir d'une part une habileté comme la capacité à exploiter les ressources de l'environnement pour parvenir à un but donné et d'autre part, en plagiant $\mathrm{N}$. Bernstein, considérer le résultat de l'apprentissage ou du développement moteur comme la maîtrise des conditions de réalisation de l'action le mouvement venant de lui même (Bersnstein, 1996, 234).

\section{Apprentissage, développement : évolutions adaptatives, interactions avec l'environnement}

L'apprentissage ou le développement serait alors un processus d'exploration et d'exploitation des différentes contraintes de la situation. Ce terme relativement général peut être précisé par deux notions, celle d'espace de la tâche et celle d'espace d'action perceptivomoteur $^{1}$. La première, utilisée notamment dans l'étude de la résolution de problème, détermine l'ensemble des états et transformations possibles d'un problème. Transposé à l'étude du comportement moteur ce concept spécifie l'ensemble des actions permises par le dispositif sur lequel on agit. Contrairement aux dispositifs expérimentaux qui ne permettent d'agir que sur une ou deux dimensions, les situations quotidiennes recèlent un nombre considérable de degrés de liberté. Elles restent ainsi plus difficiles à définir mais engendre une diversité, voire une diversification d'actions essentielle et caractéristique de la plasticité des comportements moteurs.

L'espace de la tâche est commun à tous et ne fait référence ni aux caractéristiques ni aux compétences des individus. En revanche, les caractéristiques de l'espace d'action sont déterminées par l'interaction des caractéristiques de la tâche et celles de l'individu. L'exploration, les tâtonnements consubstantiels à l'apprentissage, prennent place dans cet espace. Ces notions n'ont pour l'heure été mises en œuvre que dans des tâches expérimentales exemptes de degrés de liberté redondant (Newell 1989, 1992), mais pourraient à profit être mises en œuvres dans des tâches plus riches (Newell 1992). La nature et le nombre de degrés de liberté doivent probablement influer sur les stratégies d'exploration décrites dans ces travaux expérimentaux.

\section{Une analyse fonctionnelle de la tâche}

La définition précise de la tâche donne les premières clés de l'analyse en décrivant l'objectif du mouvement que $\mathrm{N}$. Bernstein définit comme la représentation du futur dont on a besoin (Biryukova et Bril 2002). La prise en compte du but du mouvement est également indispensable pour l'analyse. Considérer la marche avant tout comme un déplacement, peut passer pour une lapalissade, c'est pourtant à partir de cette fonctionnalité que l'on peut chercher comment sont produites les forces nécessaires au déplacement. Ainsi, contrairement à certaines tâches expérimentales trop abstraites des forces communément en jeu dans le

1 perceptual-motor workspace 
mouvement, les quatre exemples présentés ici mettent en évidence les origines multiples du mouvement et empêchent d'assimiler mouvement et contractions musculaires. Pour reprendre à nouveau les termes de N. Bernstein (Biryukova et Bril 2002), l'apprentissage ne se réduit jamais à un « formule motrice » mais consiste plutôt en une « alliance » habile avec les forces de extérieures.

Nous illustrerons la portée des principes qui viennent d'être exposés à partir de quatre exemples proches des travaux de E.J. Marey \& G. Demenÿ, montrant en quoi la compréhension de la production de mouvements finalisés ne peut faire l'économie de l'analyse des différentes contraintes qui pèsent sur la réalisation du mouvement et plus spécifiquement celles qui permettent la production des forces à l'origine du mouvement, impliquant par-là même l'étude des interactions entre l'organisme et l'environnement. Les deux premiers illustrent les interactions entre les contraintes du corps et celles de l'environnement, tandis que les deux suivants permettront d'aborder les relations avec un outil et le rôle de ces propriétés dans la construction des mouvements.

\section{Exemples d'expérimentation sur les activités finalisées}

\section{L'apprentissage de la marche}

La marche chez l'homme, comme d'ailleurs chez l'animal constitue un des domaines privilégiés des travaux de E.J. Marey et G. Demenÿ. Nous avons dénombré une dizaine de conditions expérimentales susceptibles de répondre au second objectif que E.J. Marey s'était fixé en 1883 : "chercher les conditions extérieures qui modifient les actes". Les marcheurs sont filmés et enregistrés sur des plans inclinés, dans des chemins étroits empêchant les oscillations latérales, portant des charges, chaussés de semelles de différentes raideurs, etc. G. Demenÿ dans sa présentation de la marche chez l'homme insiste sur le fait que progresser vers l'avant nécessite une force motrice par le biais d'une poussée exercée par le pied en contact avec le sol. À la suite de ses travaux avec E.J. Marey, G. Demenÿ construit un dynamographe, ancêtre des plateformes de force, qui lui permet, entre autre, de développer une problématique de la marche en terme de pression normale et tangentielle et d'accélération du centre de gravité (p. 260).

Malgré la diversité des situations de locomotions étudiées et bien qu'il existe une plaque chronophotographique célèbre figeant des premiers pas, E.J. Marey et G. Demenÿ ne semblent pas avoir consacré de travaux systématiques à la marche chez l'enfant. Dans les années qui suivirent les publication de E.J. Marey et G. Demenÿ, McGraw envisageait l'acquisition de la marche dans des termes tout à fait analogues soulignant que “... the big task ahead of the infant is to develop a resistance to and a control over the force of gravity" (1932, p. 292). À partir d'études longitudinales, en collaboration avec Y. Brenière, nous avons montré que nombre de caractéristiques comportementales des mouvements de marche du jeune enfant trouvaient une explication « simple » dès lors qu'elles étaient interprétées comme des solutions motrices permettant de créer des forces propulsives nécessaires au déplacement du corps vers l'avant (Brenière \& Bril, 1988, Bril \& Brenière, 1992, 1993). Selon cette perspective il s'agit tout d'abord de définir les conditions de productions de ces forces. En fait seul un déséquilibre produit par une distance entre le centre de gravité (CG) et le centre des pressions (CP) selon l'axe antéro-postérieur va permettre un mouvement du corps vers l'avant (voir figure 1). Dans la marche, par définition, ce déséquilibre doit être produit dans une 
succession d'appuis bipodaux et unipodaux. Au cours d'une séquence de pas le CG est positionné soit en avant du CP, ce qui correspond à une phase d'accélération du CG, soit en arrière du $\mathrm{CP}$, ce qui correspond à une phase de freinage. Si l'on considère que pour marcher vite il est plus aisé de faire des grands pas, on comprendra aisément qu'un pas long entraînera une situation de déséquilibre plus importante qu'un pas court.

\section{Insérer figure 1}

Durant les premiers mois qui suivent les premiers pas, on observe une augmentation importante de la longueur des pas, et une diminution tout aussi nette de l'écartement des pieds, les années suivantes offrant une augmentation de la longueur des pas plus progressive. Comment interpréter cette courbe de développement, une fois annulé ce qui, dans l'augmentation de la longueur de pas, pourrait être dû à la croissance de l'enfant ? L'enfant qui fait ses premiers pas n'a jamais fait l'expérience d'un déséquilibre unipodal qui, s'il n'est pas compensé par une activité posturale importante, conduira inéluctablement à une chute. La solution est alors de minimiser ce déséquilibre, de manière à ne pas tomber, tout en le produisant malgré tout puisqu'il est la condition du déplacement : des petits pas répondent dans un premier temps à cette contrainte de la tâche. Puis dès que l'enfant acquiert une certaine expérience, développe une force musculaire plus grande en particulier au niveau des abducteurs de la hanche, alors il maîtrisera mieux cette situation de déséquilibre, condition nécessaire de la marche, et pourra faire des pas plus longs.

On retrouve cette idée lorsque l'on considère le mouvement vertical du centre de gravité du corps. E.J. Marey et G. Demenÿ avaient travaillé sur la relation entre la pression des pieds au sol et le mouvement du centre de gravité ce qui permettait, à partir des courbes caractéristiques de l'accélération verticale au cours de l'appui, de spécifier l'effet de variations des contraintes externes (charges, cadence) ou interne (fatigue) (Demenÿ, 1924 : 259-268). Ici encore ces travaux ouvraient la voie à une meilleure compréhension des phases de chute et de rattrapage de l'équilibre au cours de la marche : une accélération positive résulte d'une activité de propulsion, alors qu'une valeur négative témoigne d'une situation de chute dynamique. L'accélération verticale peut donc être interprétée comme un indicateur de l'activité antigravitaire (Brenière \& Bril, 1988). Ce paramètre renseigne ainsi sur la stratégie de maintien à hauteur du centre de gravité au cours de la marche. Une analyse systématique de l'accélération verticale au cours des cinq premières années de marche chez l'enfant nous a permis de montrer que dans les premiers mois l'augmentation de longueur de pas allait de pair avec une amplification de la stratégie de chute, l'augmentation du déséquilibre n'étant pas compensée par une activité posturale. Il faut ensuite à l'enfant plusieurs années pour voir disparaître cette stratégie de chute au profit d'une stratégie de propulsion durant l'appui unipodal (Brenière \& Bril, 1998).

Un dernier aspect du processus d'acquisition de la marche, montrant le rôle des contraintes biomécaniques et celui de la gravité dans l'expression comportementale, concerne l'initiation de la marche. Lors de l'initiation d'une séquence de pas, la durée du premier pas varie d'un individu à l'autre et correspond à peu près exactement à la demi-période d'un pendule complexe inverse (Brenière et al, 1987, Brenière \& Ledebt, 1994). Cette durée varient en fonction de trois paramètres : la masse du corps, la position du centre de gravité relativement au sol et le moment d'inertie. Si l'on considère maintenant les très jeunes marcheurs, on constate que la durée de ce premier pas correspond à la demi-période d'un 
pendule inverse simple, qui elle ne dépend que d'un seul paramètre, la position du centre de gravité (Brenière et al, 1989). Ces résultats nous permettaient de proposer l'interprétation suivante : l'enfant se comporte "comme si" dans un premier temps il intégrait la position de son centre de gravité, puis dans un second temps, sa masse et son moment d'inertie.

Ces études sur la marche chez l'enfant nous ont conduit à faire l'hypothèse que l'acquisition de la capacité à marcher correspond à un apprentissage de l'utilisation des contraintes mécaniques du système composé de son corps, du sol sur lequel il marche et de la gravité ; en d'autres termes cette période est dévolue à l'intégration des contraintes posturales aux nécessités dynamiques de la marche. Cet exemple montre à quel point on ne peut faire l'économie de l'analyse du rôle des contraintes externes dans l'organisation du mouvement.

\section{Le jeu du piano}

C'est à nouveau autour de la question de relations entre mouvement et gravité qu'en 1926 N. Bernstein mène une étude sur la « bio-dynamique du touché pianistique " ${ }^{2}$. Ce travail impressionnant à de nombreux titres avait pour but premier de décrire les relations entre variations entre paramètres musicaux (tempo et intensité) et les mouvements (cinématique et dynamique). Le second objectif, plus pragmatique, traitait d'un point de technique pianistique : le rôle du poids du bras dans le jeu des octaves répétées. Nombre de théoriciens du piano considéraient que dans ce contexte musical la frappe résultait d'un mouvement passif. Plus largement, à en juger par sa critique des traités pianistiques, $\mathrm{N}$. Bernstein souhaite creuser ou plutôt démystifier par l'expérimentation, ce qui est considéré comme des mouvements naturels. Il propose ainsi à 14 pianistes virtuoses quatre tâches musicales : les deux premières consistent à jouer une série d'octaves répétées (mêmes notes) en variant l'intensité (ppp à fff) et le tempo (jusqu'à environ 8 notes par secondes), les deux suivantes permettent à travers des changements de notes et des rythmes pointés de montrer les effets d'un mouvement sur le suivant, cependant l'analyse présentée dans cet article ne concerne que les deux premières tâches.

Issue de la chronophotographie la kymocyclographique utilise à la fois une caméra rapide (520 images/sec.) et des marqueurs placés sur le corps du pianiste qui est filmé dans le plan antéro-postérieur. L'analyse cinématique des mouvements des trois articulations du bras est complétée par le calcul des moments musculaires.

L'analyse, d'une richesse étonnante, décrit notamment des changements de modes de coordination selon les contraintes de la tâche. Les tempi lents sont caractérisés par une forme de mouvement décomposée avec probablement de plus longues périodes d’inactivité musculaire, en revanche pour les tempi moyens les mouvements sont organisés en une chaîne continue. Alors que dans les tempi lents et moyens, la main et l'avant bras se meuvent sous l'effet de leurs propres forces musculaires. Dans les tempi rapides (à partir environ 6,5 notes/sec.) les mouvements de la main se transforment en une «oscillation élastique forcée ${ }^{3}$. Enfin, à l'exception des tempi extrêmement lents, moins de trois notes par secondes, les mouvement de frappe ne sont jamais dus aux forces gravitaires, ce résultat contredit donc les préceptes pianistiques évoqués précédemment. Partant du constat désormais commun de changements de régime de coordination en fonction des conditions d'exécution du mouvement, $\mathrm{N}$. Bernstein s'interroge sur la pertinence d'une technique de travail toujours abondamment utilisée par les musiciens: répéter lentement pour aller

2 “studies on the biodynamics of the piano strike” (Bernstein \& Popova 1929/2003)

3 forced elastic oscillations 
progressivement au tempo final. Cette étude du jeu du piano, singulièrement novatrice, illustre le principe que nous décrivions comme caractéristique de l'expertise. La description des oscillations forcées montre comment les pianistes experts parviennent à exploiter les contraintes de la situation, ici les propriétés de leur corps pour parvenir à leurs fins. De ce point de vue l'étude annoncée par N. Bernstein impliquant experts et débutants pourrait s'avérer particulièrement intéressante.

\section{La taille de la pierre}

L'utilisation d'outils montre de manière peut-être plus claire encore le rôle des propriétés physiques des éléments du système organisme/tâche/environnement dans la réalisation d'une action. Qu'il s'agisse de E.J. Marey et G. Demenÿ (Demenÿ, 1924) ou de N. Bernstein (1927, Biryukova \& Bril, 2002) l'utilisation d'outils tels que le marteau, la hache ou même l'épée ou la raquette de tennis (que l'on peut assimiler à un outil) ont fait l'objet de nombreuses expérimentations. Ces auteurs mettent l'accent dans leur analyse sur la vitesse de la tête du marteau ou de la pointe de l'épée, de ce que l'on désigne aujourd'hui comme le point de travail. En effet c'est bien le comportement du point de travail, plus spécifiquement sa vitesse (Ivanova, 2005), qui confère sa fonctionnalité au mouvement. G. Demenÿ dans une section intitulée "Lois de la vitesse des mouvements : rythme et masse » (1924: p. 121 et suiv.) montre, à partir de nombreux exemples, le rôle des caractéristiques des outils sur la production de la vitesse du point de travail : "la vitesse des mouvements est naturellement liée à l'importance de la masse à mouvoir et à la force des muscles moteurs. Elle est inversement proportionnelle à la masse et dépend de la force initiale et de la durée de son action. Dans un mouvement de lancer, la vitesse dépend aussi de la longueur des leviers (...) les observations faites à propos de la vitesse dans les mouvements naturels sont applicables aux mouvement avec masses additionnelles (...)» (p. 121-23). Une analyse du mouvement finalisé doit s'ancrer dans ces considérations.

\section{Insérer figure 2}

Partant de questions posées par des archéologues ${ }^{4}$, sur les caractéristiques de l'expertise impliquée dans la taille de perles de pierre dure telle qu'elle se pratique encore de nos jours à Cambay (Gujerat, Inde) (Figure2), une première expérimentation de terrain (Roux, Bril \& Dietrich, 1995; Bril, Roux \& Dietrich, 2000) avait montré que le niveau d'expertise était caractérisé par la capacité à adapter l'accélération du marteau aux contraintes locales (dureté du matériau, dimensions de l'éclat à détacher) tout en minimisant l'énergie dépensée. Par ailleurs une analyse fréquentielle de l'accélération du marteau (enregistrée à l'aide d'un accéléromètre uni-axial fixé sur la tête du marteau) montrait, chez tous les artisans, quelque soit leur niveau d'expertise, un pic de fréquence dans une fourchette relativement restreinte, entre 3,5 et 3,7 Hz. Nous avions fait l'hypothèse que ce pic de fréquence, commun à tous les artisans, rend compte des caractéristiques bio-dynamiques du couplage marteau-artisanmatière première. La capacité d'adaptation aux situations locales étant, dans cette hypothèse,

4 Cette recherche, trouve son origine dans un questionnement formulé par l'archéologue Valentine Roux, a été en partie financée par l'ACI Cognitique, et a été à l'origine de la création d'un GDR intitulé "Evaluation des habiletés techniques chez les hominidés. Habiletés impliquées dans l'action chez les Homo sapiens sapiens et chez les primates". 
due à l'aptitude du tailleur à jouer sur les propriétés de ce couplage lorsque la situation le demande.

Nous avons testé cette hypothèse par la suite en faisant varier les propriétés physiques du marteau utilisé (poids et longueur du manche). Comme l'avait très bien décrit G. Demenÿ, le pic de fréquence observé varie en fonction de la masse du marteau et de la longueur du manche (Bril et al., 2001). Les experts ont été en outre capables, malgré les modifications de leur outil, de s'adapter au mieux pour parvenir à des produits finis de bonne qualité, alors que dans ces conditions, la production des moins experts a été clairement détériorée. Ces résultats confirment à nouveau les hypothèses avancées, à savoir que l'expertise relève d'une exploitation optimale des contraintes de la situation. L'étude des coordinations du bras tenant le marteau (Biryukova et al., 2005; Biryukova et Bril, 2008) permet aussi de mettre en évidence un autre trait caractéristique d'un haut niveau de maîtrise : la possibilité de produire un effet analogue par des mouvements différents. Cette caractéristique est d'ailleurs mentionnée dans l'étude exposée précédemment (Bernstein \& Popova 1929/2003 : p. 9), à propos de la relation entre le mouvement et l'effet sonore.

\section{Le jeu du violon}

Au moment de la brouille qui opposa G. Demenÿ à E.J. Marey ses détracteurs affirmaient qu'il était tout juste bon pour jouer du violon (Manonni 1995)! Il est effectivement filmé à plusieurs reprises jouant du violon, mais il ne tirera parti de ses enregistrements qu'une quinzaine d'années après son passage à la station. Il publie en effet en 1905 un livre intitulé «le violoniste » proposant, notamment, quelques analyses de gestes techniques, ce qui était sans précédent dans l'histoire des traités sur le violon. Il y décrit, grâce à une technique astucieuse, un geste virtuose : le staccato, ce qui, au passage, laisse présager de son niveau d'expertise. S’intéressant plus spécifiquement aux mouvements du bras droit (plus facilement enregistrables) il décrit également les combinaisons de variations angulaires qui permet le mouvement rectiligne de l'archet, et plus spécifiquement le rôle du poignet dans la continuité du son lors des changements de sens de l'archet.

Le dernier exemple destiné à montrer l'interaction entre l'outil et l'utilisateur repose sur une situation spontanément expérimentale et inédite dans l'histoire de la musique: l'interprétation sur instruments anciens. Depuis l'essor de ce mouvement dans les années 1960, cohabitent pour un même répertoire, deux styles d'interprétations ainsi que deux types d'instruments. Les différences entre les violons baroques et modernes sont à la fois peu importantes, permettant donc la comparaison, mais suffisantes pour provoquer des modifications dans la manière de les utiliser. Quant aux styles d'interprétations ils sont aujourd'hui largement diffusés. Nous avons ainsi proposé à trois groupes de violonistes d'expertise différente ${ }^{5}$, de jouer le même extrait ${ }^{6}$ sur les deux instruments en imitant les deux styles d'interprétation. Demander une version à la baroque ou à la moderne permettait de fixer un objectif musical clair.

5 Des violonistes ne jouant que des instruments anciens, d'autres pratiquant fréquemment les deux instruments et à un troisième groupe de n’ayant jamais jouer de violon baroque,

6 Gavotte en Rondeau BWV 1006 de J.S.Bach 
L'analyse des enregistrements sonores reposant sur des " corrélats acoustiques » des styles d'interprétation ${ }^{7}$ a permit de montrer le bon respect de la consigne et globalement la possibilité d'imiter les deux styles d'interprétation quel que soit l'instrument (Goasdoué 2004). Une analyse plus détaillée montre cependant que l'enveloppe des sons semble plus systématiquement affectée par le changement d'instrument, alors que les variations de tempo ne changent qu'en fonction du style d'interprétation. En revanche, le simple examen des variations angulaires du coude et de l'épaule montre des modes de coordinations différenciés selon le type d'interprétation et le type d'expertise (cf. figure 3). Parmi les violonistes jouant du violon moderne (groupes 2 et 3), seuls ceux maîtrisant les deux types d'instruments sont à même de produire un mode de coordination différent selon le type d'instrument. L'explication la plus simple de ces changements consiste à prendre en compte la géométrie de la tenue du violon, en effet un simple changement dans l'angle du corps et du violon provoque de profondes réorganisations de la coordination du bras droit. Cependant, ces variations dans la tenue de l'instrument ne peuvent seules justifier ces changements. Il est probable que le mode de coordination plus propre à l'instrument baroque permette aussi de mieux maîtriser sa plus grande souplesse (raideur de l'archet et des cordes).

\section{Insérer figure 3}

Ce travail illustre à nouveau les liens étroits entre l'expertise et la maîtrise de conditions de réalisation de l'action. Il montre aussi clairement que le but, ici le projet musical, agit comme n'importe quelle contrainte sur le mouvement, comme l'ont montré $\mathrm{H}$. Winold et E. Thelen (1995) dans les mouvements des violoncellistes. Au-delà de l'adaptation aux caractéristiques de l'instrument cette expérience permet aussi de souligner la relative indépendance entre projet musical et sa réalisation, ce que $N$. Bernstein décrivait aussi dans le jeu du piano comme les différents moyens de parvenir aux mêmes fins. Enfin, cette expérience montre aussi la possibilité d'expérimenter dans un contexte musical " crédible » et de proposer une analyse compatible avec ce souci de validité écologique tel que nous l'avons définit précédemment.

\section{Pour un dialogue interdisciplinaire}

Les divergences de vues entre sciences humaines et sciences de la vie à propos du mouvement ou du geste, sont bien résumées par la distinction entre causes finales et causes efficientes. Nous proposons par le biais d'une réflexion méthodologique une voie de conciliation qui représenterait un double enrichissement. Cette conciliation qui n'est pas un compromis, pose à nouveau la prise en compte de la finalité de l'action dans l'analyse des interactions sujet-tâche-environnement. Cette forme de problème à trois corps nous paraît incontournable pour comprendre la complexité des comportements moteurs. Négliger le sens qu'a le mouvement pour celui qui l'exécute, conduit à méconnaître la manière dont il redéfinie le but qui lui est assigné par la consigne expérimentale. De la même manière l'analyse descriptive du mouvement la plus élémentaire, ne peut faire abstraction de relations entre les caractéristiques de l'individu et du contexte. Enfin, une tâche apparemment semblable peut être et être perçue comme très différentes selon les contextes.

7 Méthode d'analyse multivariée regroupant 24 critères élaborée sur la base d'enregistrements commercialisés 
Les exemples qui viennent d'être présentés illustrent clairement na nécessité de prendre en compte la finalité et le contexte pour comprendre l'organisation du comportement moteur. Nous défendons l'idée que cette prise en compte des interactions avec l'environnement n'est pas une simple précaution méthodologique, une garantie de validité écologique, mais au contraire une manière très différente d'aborder la compréhension des comportements.

Un nouvel exemple emprunté au développement moteur, souligne les implications théoriques de cette approche. La disparition vers 4 mois de la marche automatique pourtant observée à la naissance, a fait l'objet de nombreuses hypothèses théoriques sur le rôle des évolutions internes (système nerveux principalement). Or ces options maturationnistes (Gesell 1929, Forsberg, 1999) sont mises en cause par la prise en compte de l'interaction entre les propriétés du corps de l'enfant et celles de l'environnement (Thelen \& Fisher, 1982). Il suffit en effet de plonger les enfants à mis corps dans l'eau pour voir apparaître à nouveau ces mouvements alternés des jambes après leur apparente disparition. Cet argument expérimental simple et frappant est une leçon de parcimonie des explications. Ce soucis de simplicité des explications ne s'accompagne pas d'une démarche nécessairement réductionniste. Au contraire l'idée de «soft-assembled patterns » proposée par E. Thelen souligne la nature complexe et multi-déterminée des comportements ${ }^{8}$. La recherche d'abord d'explications «les plus simples » ne limite cependant pas la portée explicative et théorique de cette approche. L'idée de soft-assembly, est un concept efficace pour penser les changements que ce soit dans l'immédiat d'une situation d'action ou à l'échelle du développement (Thelen 2003).

L’étude de N. Bernstein sur le jeu du piano est un second exemple de l'apport théorique de la prise en compte du contexte et des caractéristiques des tâches. La description des changements de coordination selon les contraintes de tempo ou d'intensité préfigure nettement plusieurs principes de l'approche dynamique des coordinations aujourd'hui abondamment testés et validés. La richesse de l'analyse biomécanique lui permet de décrire les relations complexes entre contractions musculaires et mouvement : " the same scheme of muscle tensions can lead to very different patterns depending on the initial velocities and positions of the body parts participating in the movement». A cette non-univocité entre contractions et mouvement qu'il décrit grâce à l'étude du piano (Bongaardt 1997) et qu'il reprendra ensuite dans l'article sur la locomotion (1940/1967, p. 63) s'ajoute la possibilité de produire le même résultat avec des mouvements différents. Les quatre exemples cités ici illustrent clairement ce principe, d'où la nécessité de comprendre la fonctionnalité pour comprendre le mouvement. Ces constats conduisent légitimement $\mathrm{N}$. Bernstein à qualifier les mouvements humains d'écheveaux d'interactions.

\section{Apports de l'étude de l'apprentissage pour comprendre les gestes techniques}

Les décalages entre niveaux d'analyse ne sont pas seuls responsables des incompréhensions entre sciences humaines et sciences de la vie : les tâches et les méthodes employées sont déterminantes dans la complémentarité de ces approches. L'exemple de la taille de la pierre présenté ici, montre que le premier préalable pour le dialogue entre disciplines est d'étudier des activités de terrain donc de partager un objet d'étude. Ensuite, le choix d'outils d'enregistrement du mouvement impose également une définition précise des objectifs de l'observation. Cette réflexion conduit à la formulation d'hypothèses sur les

8 "Movements can be seen as 'softly-assembled' patterns created and dissolved as tasks and environments change” (Thelen 2003 b) p. 381 
principes du mouvement observé qui ne s'imposent pas aussi nettement dans l'observation à l'œil nu ou de simples films (Roux et Bril 2002). L'analyse fonctionnelle de la tâche s'avère particulièrement importante dans cette phase d'instrumentation, concevoir la marche comme un déplacement réalisé par une succession de déséquilibre conduit à s'intéresser d'abord aux modes de variations de la distance entre centre de gravité et centre des pressions au cours du pas, et non pas à la cinématique du mouvement de la jambe comme c'est la cas de nombreuses études.

Plus largement les techniques d'enregistrement permettent de redéfinir les catégories de gestes fondées sur l'apparence visuelle du mouvement et les descriptions à l'aide de notations chorégraphiques (Hewes, 1955 ; Jablonko, 1968). Un projet en cours dans notre équipe ${ }^{9}$ nous permet de discuter de la parenté fréquemment citée, et par ailleurs déterminante, dans les questions phylogénétiques entre deux gestes de percussion, la taille de la pierre et le cassage de noix (Boesch \& Boesch 1993, Sugiyama \& Koman 1979). Ce dernier exemple montre un des apports de l'expérimentation en archéologie dont pourrait bénéficier d'autres sciences humaines comme l'anthropologie ou encore la musicologie.

\section{Tâches, terrains, techniques, théories}

Les travaux de E.J. Marey et G. Demenÿ et mais également ceux de N. Bernstein illustrent l'interdépendance entre l'objet d'étude, les méthodes et les théories. La possibilité et probablement l'idée même de travailler sur des activités aussi variées n'auraient pas existé sans les techniques d'enregistrement. Inversement, la richesse des analyses biomécaniques et de leurs développements théoriques tient certainement à la richesse des tâches étudiées.

Bien que E.J. Marey, G. Demenÿ et N. Bernstein, aient été inventeurs ou au moins aient contribué à la mises au point des dispositifs d'enregistrement du mouvement, ils ne sont pas rester dans une fascination benoîte, ni pour la technique, ni pour les images qu'elles offraient. E.J. Marey que l'on peut considérer comme un pionnier du cinéma n’a, longtemps, pas reconnu d'autres intérêts que scientifiques à ses inventions, ce qui lui valu la brouille avec son préparateur (Demenÿ) qui déposa un brevet de caméra reprenant une partie de ses inventions.

Contrairement à ce que pourrait laisser envisager la profusion d'images issues de la chronophotographie et leurs qualités esthétiques, les travaux de E.J. Marey et G. Demenÿ ne se sont jamais bornés à la curiosité du ralenti ou de l'arrêt sur image. Très vite E.J. Marey et G. Demenÿ ont également compris l'intérêt de coupler différents modes d'enregistrements du mouvement (semelles exploratrices, dynamographe, odographes) et n'ont pas abandonné les apports des premiers appareils de la méthode graphique au profit de la plus spectaculaire chronophotographie. N. Bernstein bien qu'ayant considérablement sophistiqué les techniques mises au point par ses prédécesseurs français, est également très lucide sur l'apport des techniques à la compréhension du contrôle moteur. Il résume, non sans humour, son point de vue : " the idea of discovering dexterity with new, sophisticated devices is as naïve as the feelings of the simple-minded peasant who was fascinated that the astronomers had been able to discover the names of the stars by observing them through telescopes. » (Bernstein $1996 \mathrm{p}$. 20) 
Ces travaux ont également pour point commun de partir de l'activité et de n'avoir pas construit de tâches illustrant idéalement des principes théoriques. Le choix des activités analysées par E.J. Marey, comme pour nombre de théoriciens aujourd'hui célébrés (Gibson ou Bernstein), ont été souvent guidés par une demande sociale. Les promesses d'applications furent d'ailleurs l'occasion d'un débat houleux à la chambre des députés sur la chaussure du soldat, au moment où J. Ferry plaidait pour le vote d'une subvention en vue de la création de la station physiologique (Mannoni 1997, Pociello 1999). Aurait-il pu en être autrement ? Les espoirs d'application mêlés d'une foi dans le progrès ne pouvaient guère conduire à l'étude de " mouvements sans sens ». Ce climat intellectuel conduit E.J. Marey et G. Demenÿ également au choix d'une approche expérimentale, ce qui permît de montrer la possibilité et l'intérêt de réaliser des "expériences de terrain ». L’intégration du contexte et de la fonctionnalité dans l'activité est en effet indispensable à l'intelligibilité aussi bien des questions de transmission culturelle que de l'apparition de nouvelles techniques. En retour, souhaitons que ce travail sur des activités finalisées permette de repousser l'opposition récurrente et souvent implicite entre aspects moteurs et cognitifs. Pourtant comme l'écrit avec malice E. Thelen (1987) à propos d'une controverse désormais classique (Meijer, 1988) entre approches du contrôle moteur « je pense donc je bouge ${ }^{10}$.

\section{Références}

Bernstein, N. (1967). The coordination and regulation of movements. London: Pergamon Press.

Bernstein, N. A. (1996). About dexterity and its development. In M. L. Latash \& M. T. Turvey (Eds.), Dexterity and its development, with On dexterity and its development by Bernstein N.A. (pp. 1-244). Mahwah New Jersey: Lauwrence Erlbaum Associates.

Bernstein, N. A., \& Popova, T. S. (1930/2003). Studies on the Biomechanics of the Piano Strike. Motor Control, 7, 3-45.

Biryukova, E., \& Bril, B. (2002). Bernstein et le geste technique. Technologies, Idéologies, Pratiques : Revue d'Anthropologie des connaissances, 14(2), 49-68.

Biryukova, L., Bril, B. (2008). Organization of goal directed action at a high-level of motor skill: the case of stone knapping in India. Motor Control. 12, 181-209

Biryukova, E., Bril, B., Dietrich, G., Roby-Brami, A., Kulikov, M. \& Molchanov, P. (2005) The organisation of arm kinematic synergies: the case of stone-bead knapping in Khambat. Dans Stone knapping. The necessary conditions or a uniquely hominin behaviour. Sous la direction de V. Roux et B. Bril (pp.73-89), McDonald Publication: Cambridge (sous presse)

Boesch, C., \& Boesch, H. (1993). Different hand postures for pounding nuts with natural hammers by wild chimpanzees. In H. Preuschoft \& D. J. Chivers (Eds.), Hands of primates (pp. 31-43). Wien: Sringer-Verlag.

Bongaardt, R. (1997). Shifting focus: the Bernstein tradition in movement science. Free University, Amsterdam. 
Brenière, Y., \& Bril, B. (1988). Pourquoi les enfants marchent en tombant alors que les adultes tombent en marchent ? C. R. Académie des Sciences Paris, 307, 617-622.

Brenière, Y., Bril, B., \& Fontaine, R. (1989). Analysis of the Transition from Upright Stance to Steady State Locomotion in Children with Under 200 Days of Autonomous Walking. Journal of Motor Behavior, 21(1), 20-37.

Brenière, Y., Do, M. C., \& Bouisset, S. (1987). Are Dynamic Phenomena Prior to Stepping Essential to Walking? Journal of Motor Behavior., Vol.19, N⒈

Bril, B. \& Brenière, Y. (1993) Posture and gait in early childhood : learning to walk or learning dynamic equilibrium ? In G. Savelsberg (Ed.), Development of Coordination, Amsterdam: Elsevier, 337-358.

Bril, B. (2002). Apprentissage et contexte. Intellectica, 35, 251-268.

Bril, B., Dietrich, G., Byriukova, L., Roby-Brami, A. \& Roux, V. (2001). Hammering, adaptation to tool properties and raw material. Poster présenté au Workshop International "Knapping stone, a uniquely hominid behavior?". Abbaye des Premontrés, Pont-à-Mousson, 21-24 novembre.

Bril, B., \& Brenière, Y. (1992). Postural Requirements and Progression Velocity in Young Walkers. Journal of motor Behavior, Vol.24, n²1., 105-116.

Bril, B., \& Roby-Brami, A. (1994). Environnement et construction de l'action. In INSERM (Ed.), Insertion Sociale des Personnes Handicapées : Méthodologies d'Evaluation (pp. 127-135). Paris.

Bril, B., \& Roux, V. (1993). Compétences impliquées dans l'action. Le cas de la taille des perles en pierre dure (Khambat, Inde). Raisons Pratiques, 4, 267 - 286.

Bril, B., Roux, V., \& Dietrich, G. (2000). Habileté impliquées dans la taille des perles en calcédoine caractéristiques motrices et cognitives d'une action située complexe. In V. Roux (Ed.), Cornaline de l'inde, des pratiques techniques de Cambay aux technosystèmes de l'indus (pp. 207-332). Paris: éditions de la maison des sciences de l'homme.

Cole, M., (2005). Culture in development, In M. H. Bornstein, \& M. E. Lamb,. Developmental Science: An Advanced Textbook, Fifth Edition (5 ed., p. 672). Lawrence Erlbaum.

Connolly, K., \& Dalgleish, M. (1989). The emergence of a tool-using skill in infancy. Developmental Psychology, 25(6), 894-912.

Demenÿ, G. (1905). Le violoniste. Paris: Maloine.

Demenÿ, G. (1924). Mécanisme et éducation des mouvements. Paris: Alcan.

Forssberg, H. (1999). Neural control of human motor development. Current Opinion in Neurology, 9(676-682).

Gesell, A. (1929). Maturation and infant behavior pattern. Psychological Review, 36, 307319.

Goasdoué, R. (2002). L’art du violon, aperçu historique d'une pratique raisonnée. Technologies, Idéologies, Pratiques : Revue d'Anrthropologie des connaissances, 14(2), 69-93.

Goasdoué, R. (2004). Évolution des stratégies d'élaboration du projet musical et de sa réalisation dans l'apprentissage du violon. Université Paris-X, Nanterre. 
Haudricourt, A. G., 1987. La technologie science humaine. Recherches d'histoire et d'ethnologie des techniques. Paris : Editions de la Maison des Sciences de l'Homme.

Hewes, G. W. (1955). World distribution of certain postural habits. American Anthropologist, 57, 231-244.

Ivanova, G. (2005). The biomechanics of the complex coordinate stroke. Dans Dans Stone knapping. The necessary conditions or a uniquely hominin behaviour. Sous la direction de V. Roux et B. Bril (pp.119-128), McDonald Publications: Cambridge (sous presse)

Jablonko, A. (1968). A Method of Analyzing Human Movement. In Dance and Daily Activities among the Maring People of New Guinea : A Cinematographic Analysis of Body Movement Style (pp. 55-76). Columbia.

Ledebt, A., \& Breniere, Y. (1994). Dynamical implication of anatomical and mechanical parameters in gait initiation process in children. Human Movement Science, 13, 801815.

Leroi-Gourhan, A., 1964. Le geste et la parole. Technique et langage. Paris: Editions Albin Michel.

Mannoni, L. (1997). Georges Demenÿ : pionnier du cinéma. Douai: Pagine.

Marey, E. J. (1883). La station physiologique de Paris. La nature, 536, 226-234.

Mauss, M. (1936). Notion de technique du corps. Journal de Psychologie, 3-4, 365-386.

Meijer, O. G., \& Roth, K. (1988). Complex Movement Behaviour: Motor-action Controversy. North-Holland Publishing.

Newell, K. M., 1986. Constraints on the development of coordination. In M. G. Wade \& H. T. A. Withing (eds.) Motor skills acquisition, (pp. 341-60). Dortrech: Martinus Nijhoff,.

Pociello, C. (1999). La science en mouvements : Etienne Marey et Georges Demenÿ 18701920. Paris: Presses Universitaires de France.

Roux V., Bril, B. \& Dietrich G. (1995). Skills and learning difficulties involved in stone knapping: the case of stone bead knapping in Khambhat, India. World Archaeology, 27(1), 63-87.

Siguan, M. (1980). Discussion générale. In M. Richelle \& X. Seron (Eds.), L'explication en psychologie. Paris: Presses Universitaires de France.

Sugiyama, Y., \& Koman, J. (1979). Tool-using and making behavior in wild chimpanzees at Bossou, Guinea. Primates, 20, 513-524.

Thelen, E. (1987). We think; therefore, we move. Cahiers de Psychologie Cognitive, 7(2), 195-198.

Thelen, E. (2003). Connectionism and dynamic systems: are they really different? Developmental Science, 6(4), 378-391.

Thelen, E., \& Fisher, D. M. (1982). Newborn stepping: an explanation for a 'disappearing' reflex. Developmental Psychology, 18, 760-775.

Winold, H., Thelen, E., \& Ulrich, B. D. (1994). Coordination and control in the Bow Arm Movements of Highly Skilled Cellists. Ecological Psychology, 6(1), 1-31. 


\section{Légende des figures}

\section{Figure 1.}

Illustration de la trajectoire du centre des pressions (CP) et du centre de gravité (CG) au cours d'une séquence de pas.

$\Delta \mathrm{Xp}$ : longueur du pas (calculée comme la différence de position du CP dans le plan antéropostérieur, au début de l'appui unipodal, de deux pas successifs) ;

$\Delta$ Yp : largeur du pas (calculée comme la différence de position du CP dans le plan frontal, au début de l'appui unipodal, de deux pas successifs).

\section{Figure 2.}

Artisan au cours de l'expérimentation de terrain à Cambay, Gujarat, Inde. L’expérimentation s'est déroulée dans un atelier de taille qui avait été prêté pour la durée de notre séjour. Le mouvement était enregistré à l'aide de 4 capteurs éléctromagnétiques (Polhémus) et d'un accéléromètre uni-axial positionné sur le côté non utilisé du marteau.

\section{Figure 3.}

Description des changements de coordination (à partir de variations angulaires du coude et de l'épaule) lors d'une version baroque sur chaque instrument (violon baroque et violon moderne). Données pour trois violonistes.

Les "boîtes à moustaches" donnent la moyenne, l'écart type et l'amplitude des variations angulaires pour chaque articulation. 


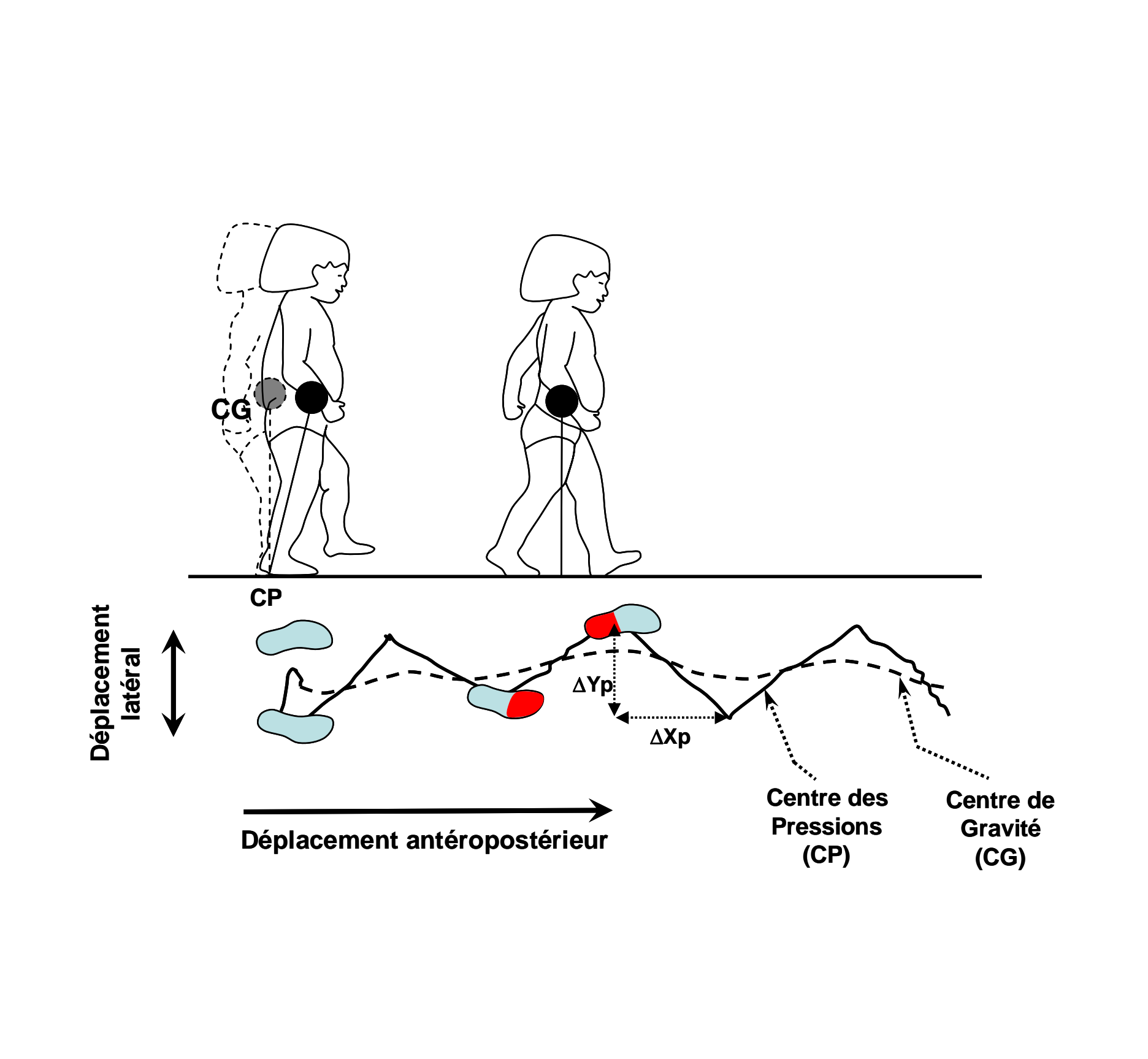




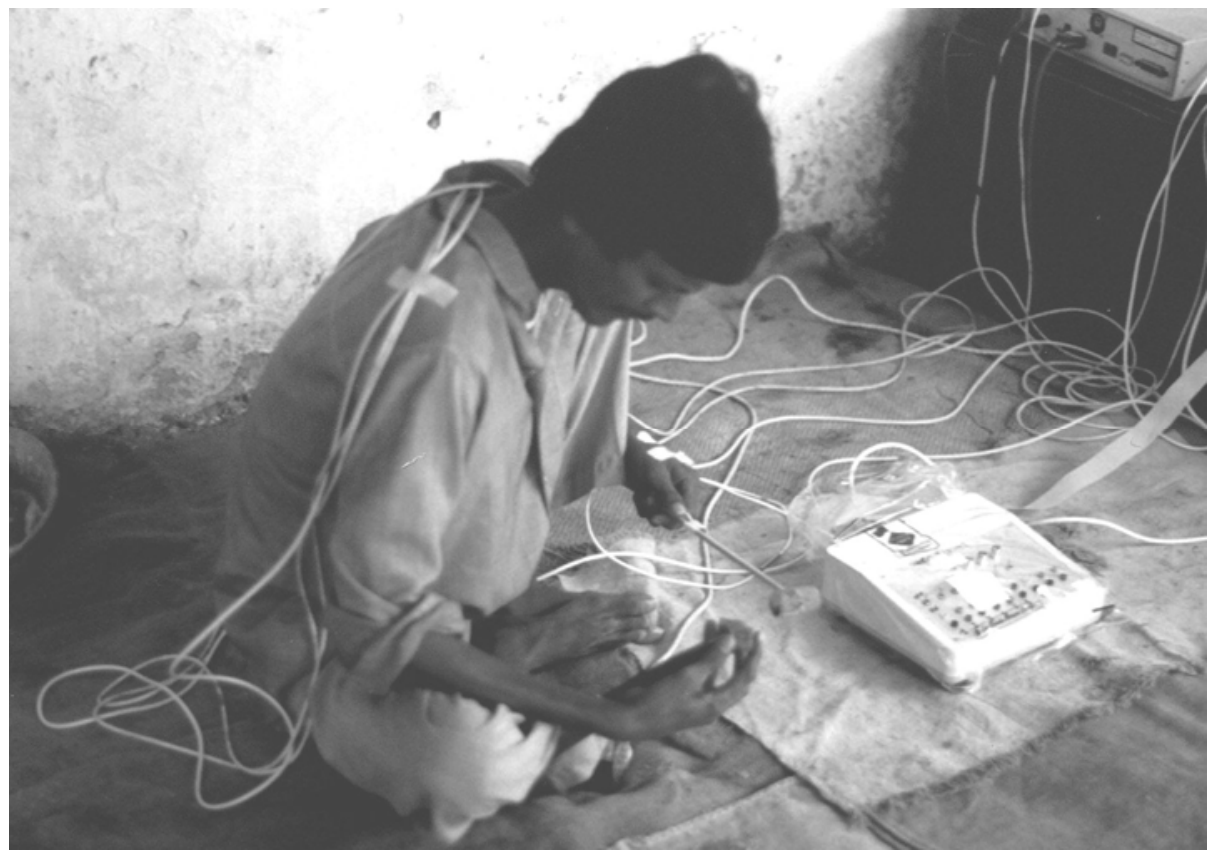


Gavotte en rondeau, BWV 1006, J.S. Bach

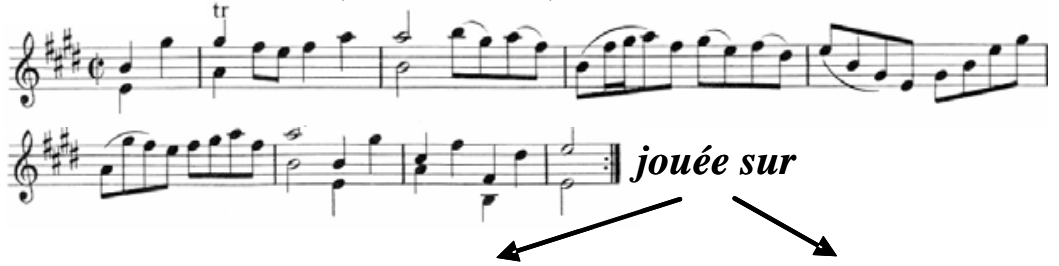

violon baroque

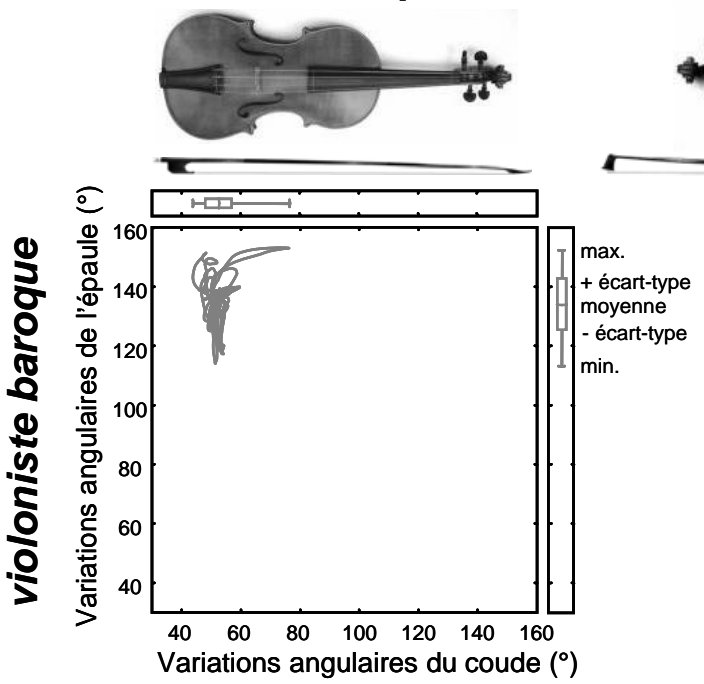

violon moderne

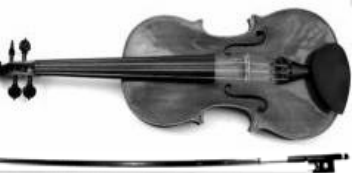

Les violonistes baroques pris à titre de groupe témoin n'ont pas joué de violon moderne
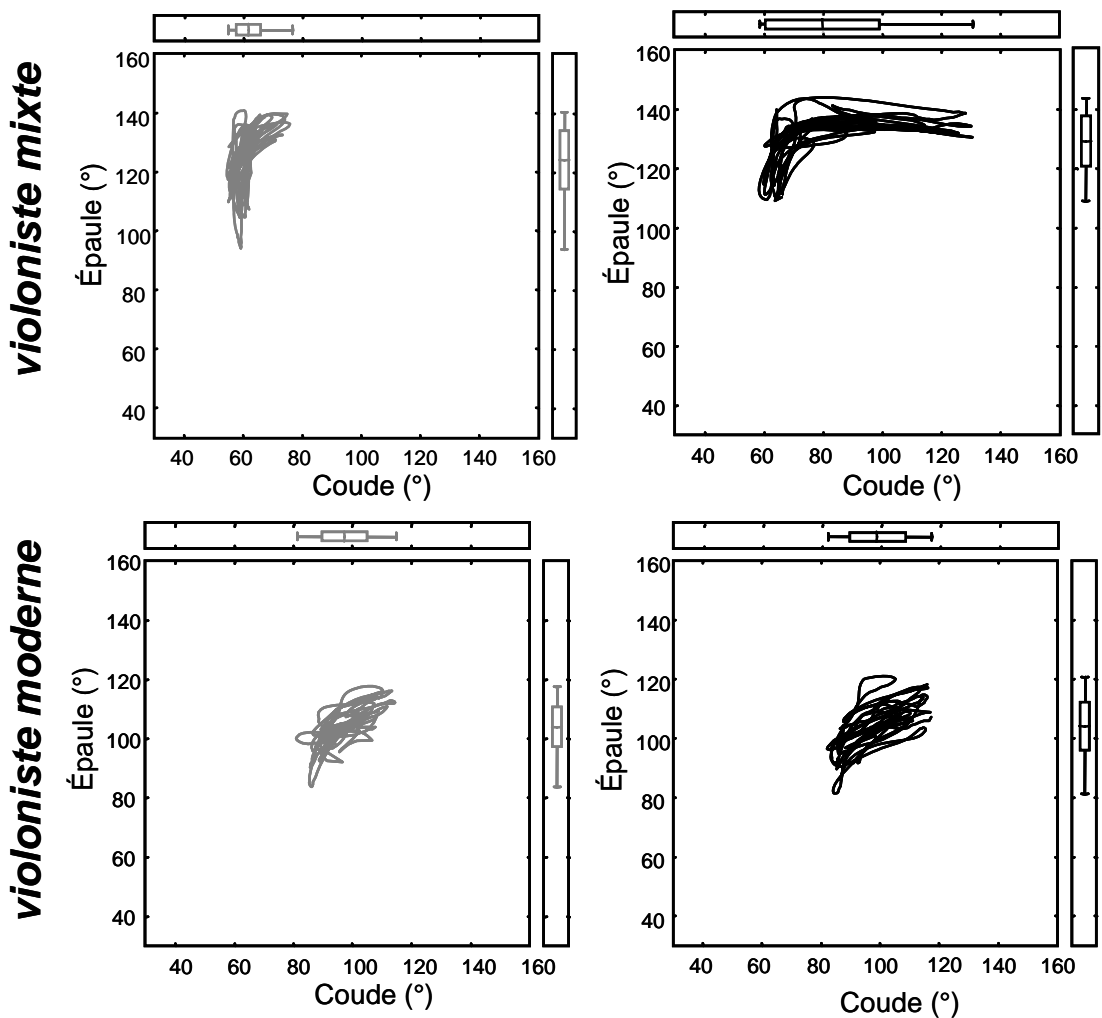